\title{
Efectos de la rehabilitación de la deglución en la producción del habla
}

\author{
Effects of rehabilitation of swallowing in speech production
}

\author{
Natalia Serrano Muñoz \\ Universidade da Coruña, España
}

\begin{abstract}
Resumen
El habla y la deglución son dos funciones que se desarrollan dentro del sistema estomatognático, por lo que comparten los mismos músculos y órganos para su ejecución. Este hecho implica la existencia de una interrelación oral y, por lo tanto, una reciprocidad en el desarrollo de las mismas. El objetivo principal del presente trabajo fue valorar los efectos que produce un programa de rehabilitación de la fase oral de la deglución en la producción del habla en un caso de traumatismo craneoencefálico grave. Los datos obtenidos mostraron una mejora significativa tanto en la propia deglución como en la articulación.

Palabras clave: neurología, caso clínico, aparato digestivo, lenguaje verbal, rehabilitación.
\end{abstract}

\begin{abstract}
Speech and swallowing are two functions that take place within the stomatognathic system, so they share the same muscles and organs for performace. This implies the existence of an oral interrelationand, therefore, reciprocity in their development. The main objective of this study was to assess the effects of a rehabilitation program of the oral phase of swallowing in speech production in a case of severe head trauma. The data showed a significant improvement in swallowing and articulation.

Keywords: neurology, clinical case, digestive system, verbal language, rehabilitation.
\end{abstract}

El traumatismo craneoencefálico (TCE) es definido como "una afectación del cerebro causada por una fuerza externa que puede producir una disminución o alteración del estado de conciencia, que conlleva una alteración de las habilidades cognitivas o del funcionamiento físico" (NHIF, citado en Junqué, Bruna, Mataró y Puyuelo, 1998).

El daño cerebral producido por un TCE implica una serie de cambios estructurales, fisiológicos y funcionales en la actividad del sistema nervioso central. Las fuerzas de aceleración-deceleración y rotacionales provocan lesiones en las estructuras encefálicas que condicionan alteraciones motoras, sensitivas, cognitivas, del lenguaje, conductuales y sensoriales (Orient-López et al., 2004).

A nivel orofacial, los músculos y las articulaciones de la región de la cabeza y el cuello también tienden a ser afectados por los agentes agresores. Cuando éstos son dañados, alteran la estructura del sistema estomatognático comprometiendo las funciones propias del mismo (Trawitzki, citado en Nascimento y Gimeniz-Paschoal, 2008).

El sistema estomatognático es la unidad orgánico-funcional en la que se integran los sistemas digestivo, respiratorio y fonoarticulatorio y donde se desarrollan, por tanto, las funciones de respiración, succión, masticación, deglución y habla (Borrás y Rosell, 2005).

La deglución es una función vital para el ser humano consistente en el transporte de alimentos sólidos y/o líquidos desde la boca hasta el estómago, atravesando la faringe y el esófago.

El proceso deglutorio se divide en tres fases; oral, faríngea y esofágica que se desarrollan sucesivamente, una a continuación de otra (Jaume y Tomás, 2007).

La fase oral constituye la primera etapa que debe recorrer el bolo antes de llegar al estómago y en ella se diferencian dos acciones cronológicas encadenadas: la preparación del bolo alimenticio y la propulsión del mismo. La fase faríngea constituye la segunda etapa del proceso deglutorio y consiste en el tránsito del bolo alimenticio a través de la faringe para llegar al esófago. Finalmente, la fase esofágica que constituye la última de las tres etapas consiste en el tránsito del bolo alimenticio desde el esófago hasta el estómago.

En relación al habla, señalar que se ejecuta mediante los órganos articulatorios. Esto es, la laringe, la faringe, el paladar blando, el paladar duro, la lengua, los dientes, las mejillas, los labios y las fosas nasales (Tanigute, 2002). La Asociación Americana de Habla, Lenguaje y Audición (ASHA) (2015) define el habla como el medio oral de comunicación, es decir, la realización concreta de la lengua en un momento y lugar determinados. Ésta se compone por tres elementos: la articulación que es la manera en que se producen los sonidos, la voz que es el uso de las cuerdas vocales y de la respiración para producir los sonidos y la fluidez que corresponde al ritmo al hablar.

En el mecanismo del habla intervienen los mismos elementos anatómicos que en la deglución; con la variante de que en esta última, los movimientos son dirigidos hacia el interior del organismo y en el habla, la energía es dirigida hacia el exterior (González, 2004).

Una correcta fase oral de la deglución precisa la integridad de la musculatura labial para asegurar el sellado labial que impide la pérdida de material por la boca, el movimiento lingual para formar y propulsar el bolo en sentido anteroposterior, la musculatura oral para evitar que el alimento quede en el vestíbulo de la cavidad bucal y el adecuado funcionamiento de la musculatura palatal (Terré-Boliart, 2002).

Por otra parte, la boca tiene una función primordial en la articulación de los sonidos, dependiendo éstos de la posición y la movilidad de la lengua, la presencia y la posición de los dientes, la movilidad de los labios y las mejillas y la posición de la mandíbula (Tanigute, 2002). 
Entre ambas funciones existe, por tanto, cierta relación práxica al compartir los mismos músculos y órganos para su ejecución. La afectación de estas estructuras a causa de las lesiones cerebrales producidas tras un TCE justifica la aparición de los trastornos de disfagia y disartria.

La disfagia neurógena se define como un trastorno de la deglución caracterizado por una dificultad en la preparación oral del bolo o en el desplazamiento del alimento desde la boca al estómago a causa de una lesión en el sistema nervioso (Terré-Boliart, 2002).

La disartria, por otro lado, se define como un trastorno del habla a consecuencia de una lesión cerebral que afecta a la expresión verbal, debido a trastornos del tono y movimiento de los músculos fonatorios (Alajouanine, 1956; Álvarez, Del Rosario Domínguez-Morales, Domínguez y Carrión, 2001; Gallardo y Gallego, 1995; Perelló, 1990).

Debido a que, como hemos comentado anteriormente, deglución y habla comparten las mismas estructuras para su ejecución, la afectación de éstas provocará dificultades en ambas funciones. No obstante, a pesar de que los músculos y los órganos alterados sean los mismos, los tratamientos que se han venido aplicando en cada una de ellas son diferentes.

Mientras que el tratamiento de la disfagia neurógena se centra en la aplicación de estrategias compensatorias y técnicas de rehabilitación (Terré-Boliart, 2002), la disartria tiene una línea de intervención basada en la rehabilitación de la respiración, la fonación, la resonancia y la articulación. Este último aspecto, en el que está centrado el presente trabajo, es tratado mediante técnicas indirectas que trabajan los movimientos orales no verbales y técnicas directas que trabajan los movimientos orales verbales a través del empleo de sonidos, sílabas y palabras (Hernández, 2007).

La efectividad de los programas de tratamiento de la disfagia neurógena queda constatada en múltiples estudios. Los resultados obtenidos tras la aplicación de estos programas, muestran la mejora de la fuerza y el control lingual (Robbins et al., 2007), la mejora de la eficacia durante el proceso deglutorio (Elmstahl, Bülow, Ekberg, Petersson y Tegner, 1999 y Hwang et al., 2007) y la reducción, por consiguiente, del tiempo de tragado (Nagaya, Kachi y Yamada, 2000). A su vez, también muestran la reducción de la frecuencia de penetraciones $\mathrm{y}$ aspiraciones laríngeas (Bogaert, Goeleven y Dejaeger, 2003).

Gran parte del éxito de los programas de tratamiento de la disfagia se debe a la aplicación de técnicas de terapia miofuncional sobre los músculos y los órganos implicados para restaurar las condiciones de tonicidad, movimiento y sensibilidad.

En este sentido, Overstake (citado en Pierce, 1980), Gommerman y Hodge (1995) y Ray (2003) realizaron estudios en los que se demostraba que la aplicación de este tipo de técnicas y procedimientos sobre los labios y la lengua mejoraba el tono muscular y el patrón deglutorio así como la articulación de los sonidos.

En una línea similar, el presente trabajo se ha centrado en la aplicación de un programa tipo de rehabilitación de la disfagia neurógena basado en la rehabilitación muscular mediante ejercicios de terapia miofuncional y la coordinación de las estructuras orofaciales mediante ejercicios funcionales con alimentos, con el fin de comprobar si la rehabilitación de la fase oral de la deglución produce mejoras concretas en la inteligibilidad del habla.

\section{Método}

\section{Participantes}

En este estudio participó una mujer de 22 años de edad que presentaba secuelas neurológicas secundarias a contusiones hemorrágicas en la región frontobasal y en los lóbulos temporales, con fractura del arco orbitario, de la pared anterior y lateral del seno maxilar, del ala del esfenoides y del arco cigomático de la mitad derecha facial; debido a un TCE grave, por accidente de tráfico, en el año 2011.

Actualmente presenta tetraparesia espástica, con disminución del rango articular, de la fuerza y trastorno del tono. Debido a esto, existe alteración en la marcha, en el equilibrio y en la coordinación; lo que provoca que no sea funcional para el desplazamiento y que necesite ayuda para la alimentación, el aseo y el vestido.

A nivel orofacial, presenta disfagia neurógena con pérdida del alimento por la boca y trastornos de la motilidad y disartria mixta.

Por último, a nivel neuropsicológico, presenta capacidad para el seguimiento de órdenes aunque sufre cambios comportamentales con episodios de agresividad y labilidad emocional.

\section{Instrumentos}

La evaluación se llevó a cabo a lo largo de dos fases. La primera correspondió con la evaluación previa a la intervención y la segunda con la evaluación posterior a la misma para la valoración de los resultados obtenidos tras el tratamiento. En ambas fases se utilizaron los siguientes instrumentos: el protocolo de exploración clínica de la deglución (Terré-Boliart, 2002) y el Test de Inteligibilidad (Monfort y Juárez, 2001).

La exploración clínica de la deglución es una prueba que proporciona al clínico evidencia indirecta de que el paciente tiene una alteración en la deglución permitiendo identificar signos y síntomas de disfagia, identificar pacientes con riesgo de aspiración, establecer la necesidad de vías alternativas de alimentación y en caso de no ser la exploración concluyente, recomendar exploraciones adicionales para establecer el diagnóstico. Según Terré-Boliart (2002), esta evaluación se puede dividir en cinco apartados principales: historia clínica, función respiratoria y relación con la deglución, exploración de la anatomía oral, exploración funcional y exploración con alimentos.

Un segundo método de evaluación empleado para registrar la evolución de la producción del habla fue el Test de Inteligibilidad (Monfort y Juárez, 2001). Se trata de una prueba destinada a registrar y medir la inteligibilidad del habla en niños o adultos con alteraciones graves. 


\section{Procedimiento}

La intervención se llevó a cabo durante un periodo de 10 semanas a lo largo de 3 fases: una primera fase de evaluación previa al tratamiento, el tratamiento propiamente dicho y una tercera fase de evaluación una vez finalizado el mismo.

La fase de evaluación previa a la intervención se realizó a lo largo de dos sesiones de 1 hora de duración cada una. En esta fase se recabaron los datos necesarios para el establecimiento de los objetivos del programa de intervención. En función de los resultados obtenidos en dicha fase, se diseñó un plan de intervención centrado en el tratamiento y rehabilitación de la fase oral de la deglución con el fin de restablecer la eficacia de la misma. Para ello, se diseñaron actividades destinadas a mejorar y rehabilitar cada aspecto de la evaluación inicial que se encontrara alterado. De esta manera, el objetivo terapéutico principal fue rehabilitar la fase oral de la deglución, para lo cual se establecieron cuatro objetivos específicos: aumentar el tono de la musculatura oral y de la lengua, aumentar la amplitud y velocidad de movimiento de mandíbula, labios y lengua, facilitar el inicio y aceleración de la deglución y coordinar las diferentes estructuras implicadas durante el proceso deglutorio.

Terminado el periodo de rehabilitación y a lo largo de una sesión se procedió a realizar una evaluación final en la que se volvieron a aplicar los apartados "exploración funcional" y "exploración con alimentos" del protocolo de exploración clínica de la deglución (Terré-Boliat, 2002) para comprobar si se produjeron cambios en el estado de la musculatura, el movimiento de las estructuras y los parámetros de eficacia durante la deglución. Además, se aplicó el Test de Inteligibilidad (Monfort y Juárez, 2001) con el fin de comprobar si la rehabilitación exclusivamente muscular influye en la mejora de la inteligibilidad del habla.

\section{Resultados}

A continuación se presenta una comparación de los resultados obtenidos, en relación a los objetivos trabajados, en la evaluación inicial y en la evaluación final de los apartados "exploración funcional" y "exploración con alimentos" del protocolo de exploración clínica de la deglución (Terré-Boliart, 2002) y del Test de Inteligibilidad (Monfort y Juárez, 2001).

Los resultados de la "exploración funcional" muestran una normalización de la tonicidad tanto en la musculatura del grupo oral como en la musculatura lingual, así como la corrección de los movimientos rotatorios de la mandíbula, de protrusión-estiramiento labial y proyección-retracción y laterales linguales. Además, han mejorado los movimientos laterales de los labios y los de elevación de la punta y el tercio lingual. No obstante, la posición de los labios en reposo sigue siendo abierta. En las tablas 1 y 2 se muestra una comparación entre los datos obtenidos en la evaluación inicial y en la evaluación realizada una vez finalizado el tratamiento en relación a la tonicidad y la movilidad facial y lingual, respectivamente.
Tabla 1.

Comparación de los resultados obtenidos en ambas fases de evaluación en relación a la musculatura oral y lingual

\begin{tabular}{lcc}
\hline Musculatura & Evaluación inicial & Evaluación final \\
\hline Grupo oral & Hipotónica & Leve hipotonía \\
Lingual & Hipotónica & Leve hipotonía \\
\hline
\end{tabular}

Tabla 2

Comparación entre los resultados obtenidos tras la exploración de la movilidad de la mandíbula, los labios y la lengua en las fases de evaluación

\begin{tabular}{|c|c|c|c|}
\hline Estructura & Movimiento & Ev. inicial & Ev. final \\
\hline \multirow{3}{*}{ Mandíbula } & Apertura-cierre & Correcto & Correcto \\
\hline & Laterales & Correcto & Correcto \\
\hline & Rotatorios & Disminuido & Correcto \\
\hline \multirow{2}{*}{ Labios } & Protrusión- & Disminuido & Correcto \\
\hline & $\begin{array}{l}\text { Laterales } \\
\end{array}$ & Disminuido & Leve dismin. \\
\hline \multirow{4}{*}{ Lengua } & Proyección & Disminuido & Correcto \\
\hline & Laterales & Disminuido & Correcto \\
\hline & Elevación punta & Disminuido & Leve dismin. \\
\hline & Elevación post. & Disminuido & Leve dismin. \\
\hline
\end{tabular}

Por otra parte, en el apartado "exploración con alimentos" se observa la mejora en los parámetros de eficacia al presentar, en la evaluación post-intervención, presencia de sellado labial, menos restos orales y 1-2 degluciones por bolo. En la tabla 3 se muestra la comparación entre la evaluación inicial y la evaluación final de los parámetros de la eficacia de la deglución.

Tabla 3.

Comparación entre la evaluación inicial y final de los parámetros de la eficacia de la deglución

\begin{tabular}{lcc}
\hline Parámetros eficacia & Ev. inicial & Ev. final \\
\hline Sellado labial & Ausencia & Presencia \\
Residuo oral & Excesivos restos & Algunos restos \\
$\mathrm{N}^{0}$ degluciones/bolo & $2-3$ & $1-2$ \\
\hline
\end{tabular}

Los resultados del Test de Inteligibilidad indican la corrección del fonema bilabial $/ \mathrm{p} / \mathrm{y}$ de los palatales $/ K /, / \check{c} / \mathrm{y} / \mathrm{n} /$ así como la mejora en la producción del fonema labiodental /f/, alveolar /s/ y velares /x/ y /k/. En la tabla 4, se comparan los resultados obtenidos en ambas fases de evaluación.

Tabla 4.

Resultados obtenidos en la producción de los fonemas en ambas fases de evaluación

\begin{tabular}{lccc}
\hline & Fonema & Ev. inicial & Ev. final \\
\hline \multirow{3}{*}{ Bilabiales } & $/ \mathrm{p} /$ & $/ \mathrm{b} /$ & Correcto \\
& $/ \mathrm{b} /$ & Correcto & Correcto \\
& $/ \mathrm{m} /$ & Correcto & Correcto \\
\hline Labiodental & $/ \mathrm{f} /$ & Omisión & Débil \\
\hline Interdental & $/ \theta /$ & Correcto & Correcto \\
\hline
\end{tabular}




\begin{tabular}{lccc}
\hline \multirow{2}{*}{ Dentales } & $/ \mathrm{t} /$ & $/ \mathrm{d} /$ & $/ \mathrm{d} /$ \\
& $/ \mathrm{d} /$ & Correcto & Correcto \\
\hline \multirow{4}{*}{ Alveolares } & $/ \mathrm{s} /$ & $/ \theta /$, omisión final & Débil \\
& $/ \mathrm{n} /$ & Correcto & Correcto \\
& $/ \mathrm{r} /$ & Correcto & Correcto \\
& & Cto., omisión & Cto., omisión \\
& $/ \mathrm{r} /$ & trabada & trabada \\
& $/ \mathrm{r} /$ & $/ \mathrm{r} /$ \\
\hline \multirow{3}{*}{ Palatales } & $/ \breve{\mathrm{c}} /$ & $/ \mathrm{i} /$ & Correcto \\
& $/ \mathrm{n} /$ & $/ \mathrm{n} /$ & Correcto \\
& $/ \mathrm{k} /$ & Omisión & Correcto \\
\hline \multirow{3}{*}{ Velares } & $/ \mathrm{g} /$ & Correcto & Correcto \\
& $/ \mathrm{x} /$ & $/ \mathrm{g} /$ & Débil \\
\hline
\end{tabular}

\section{Discusión}

El análisis de los datos obtenidos en las evaluaciones pre-intervención y post-intervención puso de manifiesto que en las variables que fueron objeto de intervención se produjeron cambios positivos entre las dos fases. Dichos cambios fueron: la mejora del tono en la musculatura oral y labial; la corrección de los movimientos rotatorios de la mandíbula, de protrusión-estiramiento labial y proyección-retracción y laterales linguales así como la mejora de los movimientos laterales de los labios y los de elevación de la punta y el tercio lingual y la mejora en los parámetros que miden la eficacia de la deglución al presentar sellado labial, menos residuos orales tras el tragado y 1-2 degluciones por bolo.

Por otra parte, la rehabilitación de la fase oral de la deglución produjo, a su vez, cambios en la producción del habla. De esta manera, tras el periodo de rehabilitación se produjeron mejoras concretas en la articulación. Éstas corresponden a la corrección del fonema bilabial $/ \mathrm{p} / \mathrm{y}$ a la de los palatales $/ K /, / \check{c} / \mathrm{y} / \mathrm{n} / ;$ y a la aproximación a la producción adecuada del fonema labiodental /f/, alveolar /s/ y velares /x/ y /k/.

Por lo tanto, se puede afirmar que el tratamiento de la disfagia neurógena influye positivamente tanto en el proceso de deglución como en la producción del habla. Estos resultados están en consonancia con los datos obtenidos en trabajos previos en los que la aplicación de este tipo de programas de rehabilitación de la disfagia mejoraba la fuerza y el control lingual así como los parámetros de eficacia deglutoria (Elmstahl, et al., 1999; Hwang et al., 2007 y Robbins et al., 2007).

En esta misma línea, Gommerman y Hodge (1995) tras haber realizado un estudio sobre la eficacia de la terapia miofuncional en la eliminación del patrón de empuje lingual durante la deglución junto con distorsión leve en la articulación, señalaban que la restitución de la tonicidad y la movilidad lingual se relaciona directamente con el habla, mejorando la producción articulatoria.

En el presente este trabajo, se ha podido comprobar que la mejora de las condiciones linguales provocó la corrección $\mathrm{o}$, en su defecto, aproximación a la producción correcta de los fonemas en los que interviene dicho órgano, esto es: los fonemas $/ \mathrm{s} /, / K /, / \breve{c} /, / \mathrm{n} /, / \mathrm{k} / \mathrm{y}$ /x/. No obstante, los resultados ponen de manifiesto que los fonemas directamente relacionados con los movimientos de elevación de la punta de la lengua (dentales y alveolares) y de elevación de su tercio posterior (velares), no están completamente normalizados; si bien su realización se aproxima a la producción correcta, todavía no se articulan adecuadamente. Estos resultados ponen de manifiesto la correlación entre tono, movimiento y ejecución ya que la mejora de las condiciones de tonicidad y movimiento de las estructuras implica la mejora en la ejecución de los patrones articulatorios.

Los resultados del presente trabajo están en la línea de los obtenidos por Ray (2003), quien llevó a cabo un estudio en el que la mejora del estado de la lengua y de los labios tras la aplicación de un programa de terapia miofuncional produjo mejoras en la inteligibilidad del habla en sujetos con problemas de articulación persistentes. En nuestro caso la mejora del tono y del movimiento labial implicó la corrección de la producción del fonema labial /p/ así como el correcto sonido del fonema labiodental /f/, el cual a término del periodo de rehabilitación únicamente necesitaba más intensidad en el soplo.

Estos datos ponen de manifiesto, una vez más, la relación existente entre habla y deglución; de tal manera que un programa no destinado, en principio, para la producción del lenguaje, mejora indirectamente el mismo. Este hecho viene avalado por investigaciones como la de Overstake (citado en Pierce, 1980), llevada a cabo con dos grupos sujetos sin daño neurológico y con dificultades de ceceo. A uno de los grupos aplicó una intervención miofuncional sobre la musculatura lingual; $\mathrm{y}$ al otro grupo terapia miofuncional más terapia del habla obteniendo resultados similares en ambos grupos. Por lo tanto, los efectos positivos del tratamiento de la disfagia sobre el habla, logrados tras la intervención miofuncional en una adulta con TCE, avalan la importancia de la rehabilitación muscular para la correcta producción de los fonemas.

Una de las limitaciones más importantes del presente trabajo tiene que ver con la duración del tratamiento. Hubiera sido recomendable llevar a cabo una intervención más prolongada que la efectuada con el fin de fortalecer las ganancias obtenidas tras la misma. Asimismo, deberían realizarse sesiones de seguimiento para confirmar si los logros se mantienen en el tiempo. Por otra parte, además de la evaluación de la articulación, el trabajo debería contar con una evaluación más exhaustiva de los otros elementos del habla, voz y fluidez, para comprobar si también se producen cambios en ellos.

Igualmente, otra posible línea de investigación consistiría en aplicar un programa de rehabilitación de la deglución en un caso de disfagia orofaríngea para conocer qué mejoras relacionadas con el habla se producen únicamente con la rehabilitación de la fase oral y cuáles se producirían tras la intervención de la fase faríngea, estableciendo de la misma manera, una correlación entre esta última fase y el habla. 


\section{Referencias}

Alajouanine, T. (1956). Verbal realization in aphasia. Brain, 79, 1-28.

Álvarez, F. V., del Rosario Domínguez-Morales, M., Domínguez, O. V. y Carrión, J. L. (2001). Disartria espástica: rehabilitación de la fonación de un paciente con traumatismo cráneo-encefálico. Revista española de neuropsicología, 3(4), 34-45.

American Speech-Language-Hearing Association. (2015). ¿Qué es el lenguaje? ¿Qué es el habla?. Recuperado el 08 de Junio de 2015: http://www.asha.org/public/speech/development/Quees-el-Lenguaje/

Bogaert, E., Goeleven, A., y Dejaeger, E. (2003). Effectmeting van therapeutische interventies tijdens radiologisch slikonderzoek. Tijdschrift voor Geneeskunde, 59(22), 1410-1414

Borrás, S. y Rosell, V. (2005). Guía para la reeducación de la deglución atípica y trastornos asociados. Valencia: Nau Llibres.

Elmståhl, S., Bülow, M., Ekberg, O., Petersson, M., y Tegner, H. (1999). Treatment of dysphagia improves nutritional conditions in stroke patients. Dysphagia, 14(2), 61-66.

Gallardo, J. R. y Gallego, J. L. (1995). Manual de logopedia escolar. Málaga: Aljibe.

Gommerman, S. L., y Hodge, M. M. (1995). Effects of oral myofunctional therapy on swallowing and sibilant production. The International journal of orofacial myology: official publication of the International Association of Orofacial Myology, 21, 9-22.

González, M. C. (2004). El aparato bucal y su relación con las regiones de la cara. Desarrollo, estructura y función. Caracas: UCV, Consejo de Desarrollo Científico y Humanístico.

Hwang, C. H., Choi, K. H., Ko, Y. S., \& Leem, C. M. (2007). Pre-emptive swallowing stimulation in long-term intubated patients. Clinical rehabilitation, 21(1), 41-46.

Hernández, N. M. (2007). Intervención logopédica en la disartria. Revista de Logopedia, Foniatría y Audiología, 27(4), 187-197.

Jaume, G. y Tomás, M. (2007). Manejo de la disfagia y aspiración. Madrid: Ergón Creación.

Junqué, C., Bruna, O., Mataró, M. y Puyuelo, M. (1998). Traumatismos craneoencefálicos. Un enfoque desde la Neuropsicología y la Logopedia. Guía práctica para profesionales y familiares. Barcelona: Masson.

Monfort, M., y Juárez, A. (2001). Test de inteligibilidad. Madrid: Entha.

Nagaya, M., Kachi, T., y Yamada, T. (2000). Effect of swallowing training on swallowing disorders in Parkinson's disease. Scandinavian journal of rehabilitation medicine, 32(1), 11-15.

Nascimiento, E. N. y Gimeniz-Paschoal, S. R. (2008). Os acidentes humanos e suas implicações fonoaudiológicas: opiniões de docentes e discentes sobre a formação superior. Ciênc Saúde Coletiva, 13(2), 2289-98.

Orient-López, F., Sevilla-Hernández, E. Guevara-Espinosa, D., Terré-Boliart, R.,
Ramón-Rona, S. y Bernabeu-Guitart, M. (2004). Resultado funcional al alta de los traumatismos craneoencefálicos graves ingresados en una unidad de daño cerebral. Revista de Neurología, 39(10), 901-6.

Perelló, J. (1990). Trastornos del habla. Barcelona: Masson.

Pierce, R. B. (1980). The role of myofunctional therapy in speech pathology. The International journal of oral myology, 6(4), 11-13.

Ray, J. (2003). Effects of orofacial myofunctional therapy on speech intelligibility in individuals with persistent articulatory impairments. The International journal of orofacial myology: official publication of the International Association of Orofacial Myology, 29, 5-14.

Robbins, J., Kays, S. A., Gangnon, R. E., Hind, J. A., Hewitt, A. L., Gentry, L. R., y Taylor, A. J. (2007). The effects of lingual exercise in stroke patients with dysphagia. Archives of physical medicine and rehabilitation, 88(2), 150-158.

Tanigute, C. C. (2002). Desarrollo de las funciones estomatognáticas. En I. Q. Marchesan. (Ed.), Fundamentos de Fonoaudiología. Aspectos clínicos de la motricidad oral (pp. 1-6). Madrid: Panamericana.

Terré-Boliart, R. (2002). Diagnóstico de la disfagia neurógena: exploración clínica. En M. Bernabeu. (Ed.), Disfagia neurógena: evaluación y tratamiento (pp. 37-51). Badalona: Fundació Institut Guttmann. 